\title{
The Detector Design of the Jefferson Lab EIC
}

\author{
Markus Diefenthaler*t \\ Jefferson Lab \\ E-mail: mdiefentejlab.org
}

The Electron-Ion Collider (EIC) is envisioned as the next-generation U.S. facility to study quarks and gluons in strongly interacting matter. The broad physics program of the EIC aims to precisely image gluons in nucleons and nuclei and to reveal the origin of the nucleon spin by colliding polarized electrons with polarized protons, polarized light ions, and heavy nuclei at high luminosity. The Jefferson Lab EIC (JLEIC) design is based on a figure-8 shaped ring-ring collider. The luminosity, exceeding $10^{33} \mathrm{~cm}^{-2} \mathrm{~s}^{-1}$ in a broad range of the center-of-mass energy and maximum luminosity above $10^{34} \mathrm{~cm}^{-2} \mathrm{~s}^{-1}$, is achieved by high-rate collisions of short small-emittance lowcharge bunches made possible by high-energy electron cooling of the ion beam and synchrotron radiation damping of the electron beam. The polarization of light ion species ( $\mathrm{p}, \mathrm{d},{ }^{3} \mathrm{He}$ ) can be easily preserved and manipulated due to the unique figure- 8 shape of the collider rings. The focus of this presentation is put on the JLEIC primary detector that has been designed to support the full physics program of the EIC and to provide essentially full acceptance to all fragments produced in collisions. The detector has been fully integrated with the accelerator and extended to the forward electron and hadron regions to achieve exceptional small-angle acceptance and resolution as well as high-precision electron polarimetry and low- $Q^{2}$ tagging. The Central Detector design allows for excellent tracking up to small angles and excellent hadron PID resulting and offers a great performance, in particular for semi-inclusive and exclusive measurements. The combination of high luminosity, highly polarized lepton and ion beams, and a full acceptance, multi-purpose detector fully integrated with the accelerator will allow JLEIC a unique opportunity to make breakthroughs in the investigation of the strong interaction.

XXIV International Workshop on Deep-Inelastic Scattering and Related Subjects

11-15 April, 2016

DESY Hamburg, Germany

\footnotetext{
* Speaker.

${ }^{\dagger}$ On behalf of the JLEIC Detector and Interaction Region Study Group.
} 


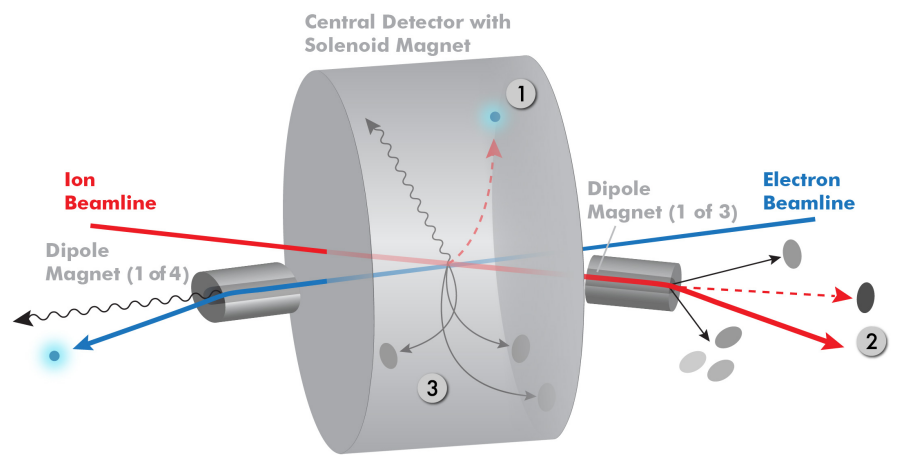

Figure 1: JLEIC IR and detector concept illustrating the crossing angle between the electron and ion beams and the dipole magnets downstream of the Central Detector.

\section{Note on the proceedings}

The following text is an excerpt of the Jefferson Lab Electron-Ion Collider (JLEIC) documentation on the interaction region and detector design [1].

\section{Physics Processes at the EIC and Acceptance requirements}

The basic physics process at the Electron-Ion Collider (EIC) is deep inelastic scattering (DIS). We can, qualitatively, define three classes of particles in the final state:

1. The scattered electron,

2. Particles associated with the initial state ion,

3. Particles associated with the struck parton.

The aim of the EIC is the investigation of the QCD structure of hadrons including ions and nucleons. All three types of final state particles carry information about the ion. Therefore it is essential that the interaction region (IR) and the detector at the EIC are designed so that all three types of particles are measured at as close to $100 \%$ acceptance as possible and with the necessary resolutions. We define the concept of a total acceptance detector as one that achieves close to $100 \%$ acceptance.

\section{Total Acceptance Detector}

The difficulty in achieving good acceptance in the forward regions at a collider has to do with the accelerator elements needed to deliver the colliding beams. To first order, the luminosity at the IP is inversely proportional to the distance between the last upstream and first downstream final focus quadrupoles (FFQs). Thus, the statistical uncertainty of measurements at the Central 


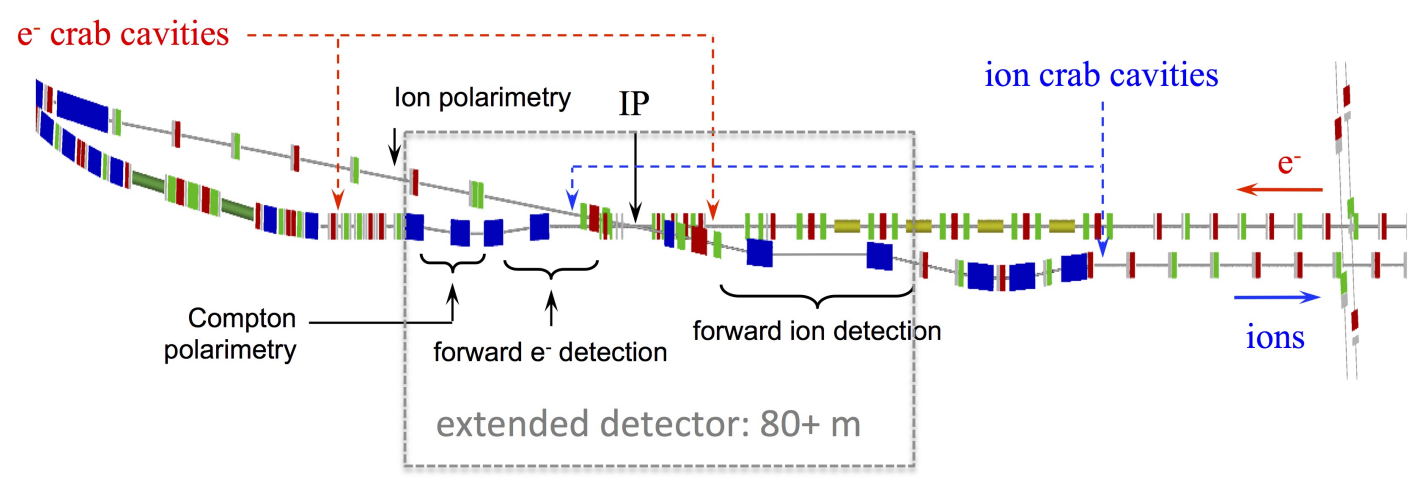

Figure 2: JLEIC IR and Extended Detector

Detector scales as $\sqrt{\text { distance. }}$. On the other hand, the closer the beam elements are to the IP, the more they obstruct the acceptance at shallow angles with respect to the beam axis and restrict the acceptance for forward particles. Also, the solenoidal field used in the Central Detector region to measure the high $P_{t}$ particles in the Central Detector is not effective in determining the momenta of particles moving parallel to the beam direction, and additional fields are needed.

The Jefferson Lab EIC (JLEIC) IR and detector concept, shown in Fig. 1, is designed to overcome the measurement difficulties posed by the beam elements. The ion and electron beams cross at a relatively large angle of 50mrad at the IP. High luminosity is preserved through the use of crab cavities. This angle moves the ion beam away from the electron beam elements and makes room for dipoles located just downstream of the Central Detector area. The dipoles serve two purposes. First, they shape the ion beam orbit so that there is $1 \mathrm{~m}$ distance between the two beams $30 \mathrm{~m}$ away from the IP, making room for detectors. Second, the dipole systems allow momentum analysis of the particles with small transverse momentum with respect to the beams. The particles with large transverse momenta are analyzed using the solenoidal field in the Central Detector. The JLEIC design and the extended detector are shown in Fig. 2. The Central Detector is about $12.5 \mathrm{~m}$ in length and is a compromise between luminosity and space considerations for the detectors.

The detection regions in the electron and ion beam directions extend $30-40 \mathrm{~m}$ in either direction from the Central Detector. In the next section, the requirements and design for the Central Detector are discussed. The far-forward hadron detector and far-forward electron detector regions are described in Rik Yoshida's proceedings on "JLEIC forward detector design and performance".

\section{Central Detector Region}

The Central Detector is designed mainly to measure those final state particles from the hard collision between the electron and the parton in the ion (Particles of types 1 and 3 in Fig. 1) and is very much like the traditional collider detectors.

The basic kinematic variables of DIS are $Q^{2}$, the virtuality of the exchanged photon, $x$, the fraction of the longitudinal momentum carried by the struck parton, and $y$, the inelasticity of the collision. The three variables are related by $Q^{2}=s x y$, where $s$ is the center-of-mass energy of the 


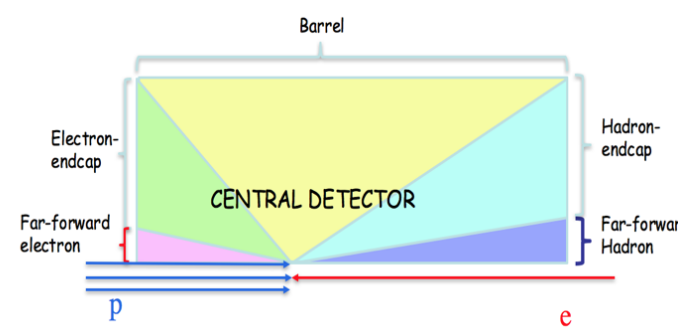

Figure 3: Regions in the JLEIC Central Detector.

collision. Thus, measuring any two of $x, y$, or $Q^{2}$, specifies the longitudinal kinematics of DIS completely. While this measurement of two variables (taken to be $x$ and $Q^{2}$ ) is not enough for much of the physics program at the EIC, they need to be measured for almost all events to be used for physics analyses.

The scattered electron energy $E_{e}$ and the scattering angle $\theta_{e}$ can be used to reconstruct the variables, $x$ and $Q^{2}$. The angle of the scattered parton and its energy (substituted with the angle and energy of the associated jet, $\theta_{\text {jet }}$ and $E_{\text {jet }}$ ) can also be used. Conversely, specifying $x$ and $Q^{2}$ completely determines the four measureable variables, $e$, jet, $E e$, and $E_{\text {jet }}$. Therefore, the energy of the scattered electron or jet that point towards any particular region of the Central Detector (i.e. fixed $\theta$ ) is predicted from simple kinematics and can be taken into account in detector design.

Access to the transverse kinematics (transverse momentum $k_{t}$ and impact parameter $b_{t}$ ), beyond $x$ and $Q^{2}$, as well as flavor identification of the partonic collision is central to the nucleon and nuclear structure measurement program planned for the EIC. The energy scale of transverse kinematics is in the order of $\Lambda_{\mathrm{QCD}}(\approx 200 \mathrm{MeV})$. This means identification and precise measurements of single hadrons among the particles associated with the scattered partons (Particles of type
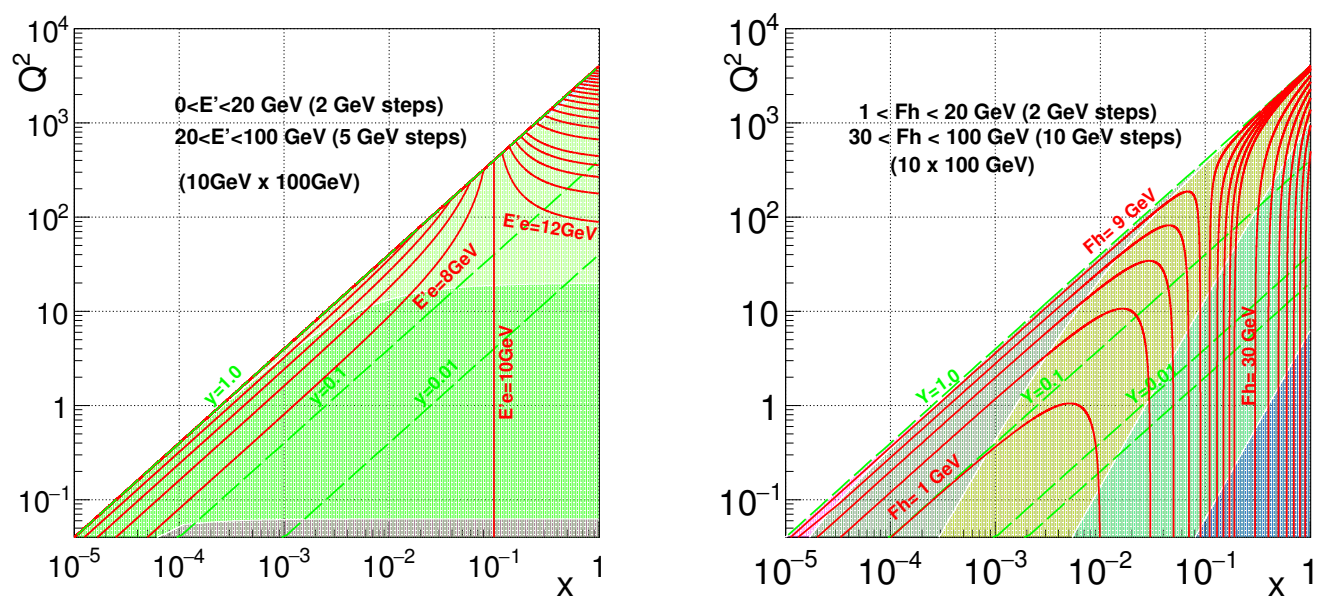

Figure 4: Isolins of the scattered electron (left panel) and jet (right panel) in the JLEIC Central Detector. 
3 in Fig. 1) are needed. In order to determine the detector requirements, the momentum range of these particles in various parts of the Central Detector must be understood. This follows from understanding of the jet energy that is expected for a fixed $\theta_{\text {jet }}$ and the momentum distribution of particles within that jet.

The Central Detector is divided into three sections, the Electron-endcap, the Hadron-endcap and the Barrel as shown in Fig. 3. Far-forward sections (both electron and hadron) will be discussed separately in Rik Yoshida's proceedings on "JLEIC forward detector design and performance". The three different Central Detector sections that correspond to different $x$ and $Q^{2}$ regions for the scattered electron are shown in the left panel of Fig. 4 along with the lines of constant scattered electron energy $E_{e}$. The same for the jet energies $E_{\text {jet }}$ is shown in the right panel of Fig. 4.

From these figures, the range of energies for $E_{e}$ and $E_{\text {jet }}$ in each section of the detector can be read. Using Monte Carlo simulation the momentum range of hadrons that correspond to particles in each detector section can be estimated. The following table gives, then, a rough estimate of the momenta for each of type of particle in each section.

\begin{tabular}{llll}
\hline & Electron-Endcap & Barrel & Hadron-Endcap \\
\hline$E_{e}$ & $<8 \mathrm{GeV}$ & $8-50 \mathrm{GeV}$ & $>50 \mathrm{GeV}$ \\
$E_{\text {jet }}$ & $<10 \mathrm{GeV}$ & $10-50 \mathrm{GeV}$ & $20-100 \mathrm{GeV}$ \\
$E_{\text {hadrons }}$ & $<10 \mathrm{GeV}$ & $<15 \mathrm{GeV}$ & $15-50 \mathrm{GeV}$ \\
\hline
\end{tabular}

These energy ranges inform the choice of technologies needed in the Central Detector for tracking, calorimetry and particle identification. The current JLEIC Central Detector design is shown in Fig. 5.

\section{References}

[1] JLEIC Detector and Interaction Region Study Group, Jefferson Lab Electron-Ion Collider (JLEIC): An Introduction to the Interaction Region and Detector Design, JLEIC Documentation Series 001, 2016

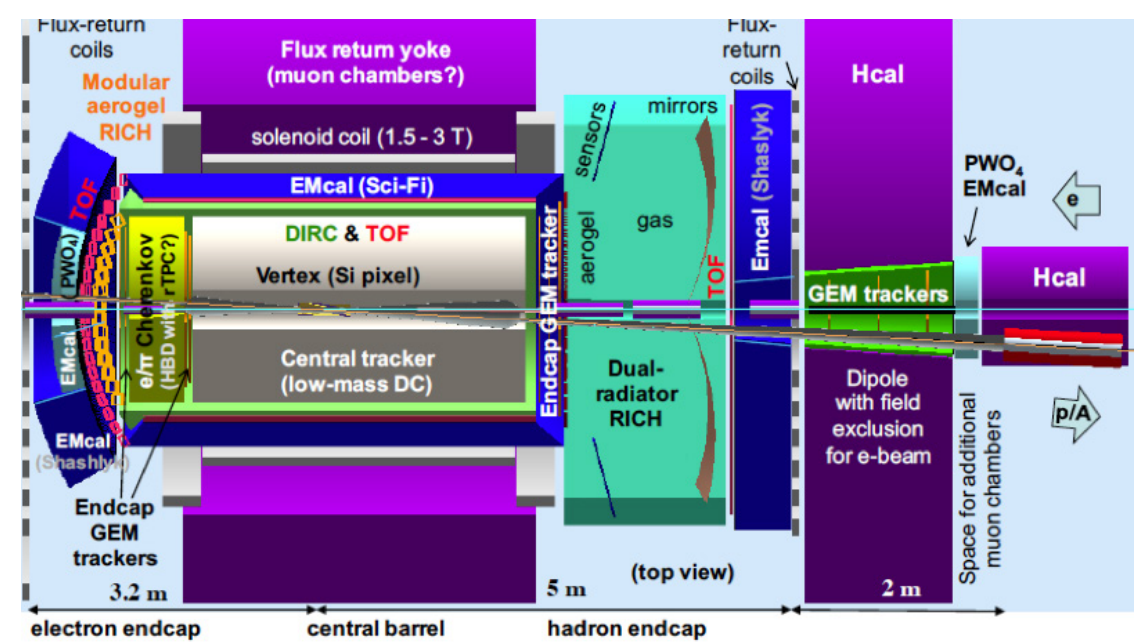

Figure 5: Detector concept for the JLEIC Central Detector 